\title{
VIRTUALIZACIÓN, UNA SOLUCIÓN PARA LA EFICIENCIA, SEGURIDAD Y ADMINISTRACIÓN DE INTRANETS
}

\author{
Diego Martín, Mónica Marrero, Julián Urbano, Eduardo Barra y José-Antonio \\ Moreiro
}

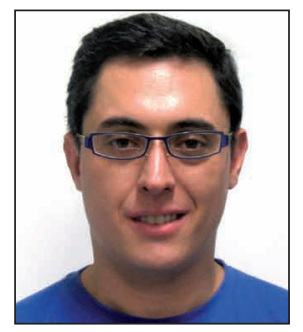

Diego Martín es ingeniero en informática por la Universidad Carlos III de Madrid, donde actualmente está realizando su tesis doctoral en ciencia y tecnología informática. Desde 2006 es profesor ayudante en el Departamento de Informática de dicha universidad, con docencia en ingeniería informática y grado en informática sobre temas relacionados con modelado e ingeniería de la información, análisis y diseño de software, arquitectura de sistemas y procesos del software.

$$
\begin{array}{r}
\text { Universidad Carlos III de Madrid } \\
\text { Departamento de Informática } \\
\text { Edificio Sabatini } \\
\text { Avda. Universidad, 30. } 28911 \text { Leganés (Madrid) } \\
\text { dmandres@inf.uc3m.es }
\end{array}
$$

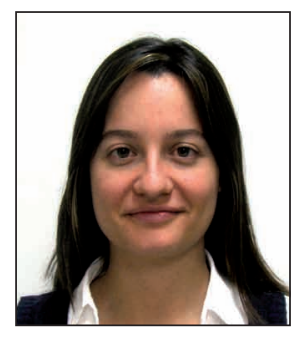

Mónica Marrero es ingeniera técnica en informática y licenciada en documentación. Ha realizado el máster en ciencia y tecnología informática en la Universidad Carlos III de Madrid y trabaja como profesora ayudante en el Departamento de Informática de la misma universidad, mientras realiza la tesis doctoral. Aunque ha realizado investigaciones sobre ingeniería del software, su principal área de interés es la extracción y recuperación de información, tanto desde un punto de vista investigador como docente.

Universidad Carlos III de Madrid Departamento de Informática Edificio Sabatini

Avda. Universidad, 30. 28911 Leganés (Madrid) mmarrero@inf.uc3m.es

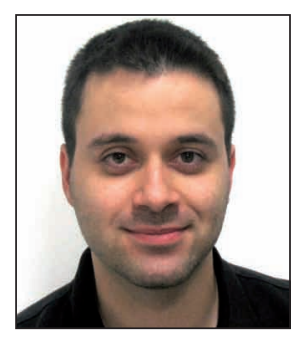

Julián Urbano es ingeniero bilingüe en ingeniería informática y ha realizado un máster en ciencia y tecnología informática. Tras una beca como ayudante de investigación en Virginia Tech (EUA), actualmente es profesor ayudante del Departamento de Informática de la Universidad Carlos III de Madrid, donde cursa el doctorado en ciencia y tecnología informática. Sus principales áreas de investigación son la evaluación de sistemas de recuperación de información y la recuperación de información musical.

Universidad Carlos III de Madrid Departamento de Informática Edificio Sabatini Avda. Universidad, 30. 28911 Leganés (Madrid) jurbano@inf.uc3m.es

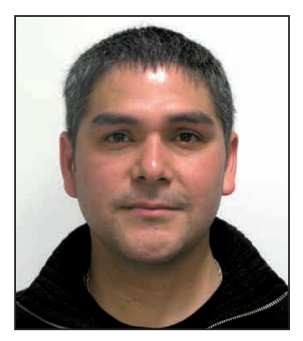

Eduardo Barra es ingeniero en mecánica-eléctrica por la Universidad Veracruzana (México), homologado al grado de ingeniero en España. Es estudiante de doctorado en ciencia y tecnología informática en la Universidad Carlos III de Madrid, donde es además profesor ayudante de ingeniería del software en el Departamento de Informática. Sus principales áreas de investigación están relacionadas con la ingeniería de software. Apoyado en el paradigma orientado a aspectos propone técnicas para la organización de la información e inferencia de conocimiento.

$$
\begin{array}{r}
\text { Universidad Carlos III de Madrid } \\
\text { Departamento de Informática } \\
\text { Edificio Sabatini } \\
\text { Avda. Universidad, 30. } 28911 \text { Leganés (Madrid) } \\
\text { ebarra@inf.uc3m.es }
\end{array}
$$

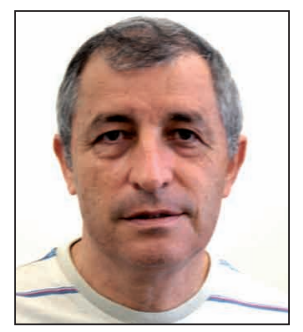

José-Antonio Moreiro es catedrático de biblioteconomía y documentación de la Universidad Carlos III de Madrid. Es autor de trabajos sobre técnicas de análisis de contenido documental y cuestiones conceptuales de la documentación. Ha sido director del Departamento de Biblioteconomía y Documentación y decano de la Facultad de Humanidades, Comunicación y Documentación de la misma universidad.

Universidad Carlos III de Madrid Departamento de Biblioteconomía y Documentación C/ Madrid, 126. 28903 Getafe (Madrid) jamore@bib.uc3m.es 


\title{
Resumen
}

La virtualización es una tecnología que permite abstraer la parte software de una computadora y desplegarla fácilmente en otra donde puede haber albergadas más máquinas virtualizadas. Este mecanismo nos permite alojar más de una computadora virtual en una computadora física. Una intranet está formada por un conjunto de computadoras que funcionan como servidores y que en la mayor parte de los casos están infrautilizadas. Se muestra la virtualización como una herramienta que nos ayuda a tener una intranet más eficiente al reducir el número de computadoras en ella; más segura porque permite la realización de copias de seguridad de forma automatizada y más fácil de administrar porque centralizamos nuestros servicios en unos pocos servidores. Todo esto hace que la virtualización nos permita ahorrar en hardware, electricidad y mantenimiento.

\section{Palabras clave}

Virtualización, Intranet, Internet, Servicios web, Máquinas virtuales.

\section{Title: Virtualization: a solution for efficiency, security and management of intranets}

\begin{abstract}
Virtualization is a technology that permits the abstraction of the software from one computer and its easy deployment in another one, which may house additional virtualized machines. This mechanism allows us to host more than one virtual computer in just one actual computer. An intranet is made up of a set of computers running as servers that are underutilized for the most part. In this paper we want to show virtualization as a tool allowing us to have a more efficient intranet because it helps us reduce the number of computers in the net; more secure because it allows the creation of backup copies automatically; and easier to manage because we centralize all our services with a handful of servers. With all this, virtualization allows hardware, electricity and maintenance savings.
\end{abstract}

\section{Keywords}

Virtualization, Intranet, Internet, Web services, Virtual machine.

Martín, Diego; Marrero, Mónica; Urbano, Julián; Barra, Eduardo; Moreiro, José-Antonio. "Virtualización, una solución para la eficiencia, seguridad y administración de intranets.". El profesional de la información, 2011, mayo-junio, v. 20, n. 3, pp. 348-354

DOI: 10.3145/epi.2011.may.16

\section{Introducción}

La virtualización ha sido identificada (Gartner Inc., 2010) como una de las diez tecnologías estratégicas para 2010 Consiste en la extracción del software de una computadora, encapsulándolo en algo que llamaremos máquina virtual, que será ejecutada en una máquina física ajena a la anterior. Aplicado a una red interna de servicios de una organización logramos pasar de una intranet con varios servidores infrautilizados a otra con unos pocos servidores ofreciendo los mismos servicios.

Las ventajas de la virtualización son muchas, las más evidentes son el ahorro en hardware, normalmente en una relación de 10 a 1 servidores en el caso más optimista, y de 6 a 1 en el caso más pesimista (VMWare Inc., 2010). Esto conlleva reducciones en costes de mantenimiento y administración de la intranet, además de reducciones en el consumo eléctrico. Se podría llegar a ahorrar hasta $7.000 \mathrm{KWh} /$ año por servidor (lo que supone $1.500 €$ anuales) (VMWare Inc., 2008a). El ahorro energético es un aspecto a tener en cuenta ya que existen informes (Fundación Vida Sostenible, 2010) que confirman que la tendencia del precio de la energía va a ser creciente. Además, nos ofrece la posibilidad de alinearnos con políticas medioambientales, ya que si más del $50 \%$ de la energía eléctrica en España procede de combustibles fósiles (REE, 2010), al reducir el consumo eléctrico se reducirán las emisiones de dióxido de carbono a la atmósfera. Se podrían dejar de emitir 4 toneladas de dióxido de carbono al año por servidor virtualizado, el equivalente a quitar 1,5 coches de la carretera (VMware Inc., 2010).

La virtualización también ofrece ventajas en la seguridad y movilidad de los sistemas. La realización de copias de seguridad es un servicio prestado por cualquier software de virtualización que, además, al permitir copias de la máquina virtual completa, facilita su traslado a nuevas ubicaciones y su puesta a punto inmediata.

La virtualización es la abstracción de los recursos de una computadora y su puesta en funcionamiento como máquina virtual en otra máquina física

En este artículo definiremos en mayor detalle qué es la virtualización y cuáles son los tipos principales, para posteriormente explorar sus ventajas e inconvenientes al aplicar esta tecnología a servicios sobre intranets. Finalizaremos exponiendo casos de éxito y conclusiones acerca de esta tecnología. 


\section{¿Qué es la virtualización?}

Es la abstracción de los recursos de una computadora (Turban et al., 2008) y su puesta en funcionamiento como máquina virtual en otra máquina física. El término "máquina anfitriona" se refiere a la máquina física donde se lleva a cabo la virtualización mientras que el término "máquina huésped" se refiere a la máquina virtual (también llamada hypervisor o virtual machine monitor - VMM).

Un sistema de virtualización debe ser capaz de ofrecer una interfaz en la máquina anfitriona para poder interactuar con el sistema operativo de la máquina virtual. Además, la máquina anfitriona debe ofrecer una interfaz de sus recursos a la máquina virtual para que pueda utilizarlos. De estas interfaces de comunicación se encarga un software que se instala en la máquina anfitriona para poder ejecutar las máquinas virtuales. Existen muchos tipos de software de virtualización en el mercado, tanto software propietario como software libre y para ejecutarse sobre máquinas anfitrionas que funcionen como servidores, o bien sobre cualquier computadora personal. Entre los más utilizados están VirtualBox y Virtual PC para computadoras personales, Xen y KVM para servidor, y VMware para ambos.

\section{Virtualización de hardware}

Se llama también virtualización de plataforma (Popek; Goldberg, 1974) y se refiere a la creación de máquinas virtuales que se comportan como máquinas reales con su propio sistema operativo, funcionando en una máquina anfitrión que carece de sistema operativo. La máquina anfitriona sólo ejecuta un software de virtualización para poder ejecutar las máquinas virtuales (ver figura 1). Esta suele ser la opción más utilizada cuando tenemos varios servidores, ya sean servidores de email, impresoras en red, ficheros, gestores de contenido, etc. y queremos hacerlos funcionar en una sola máquina.

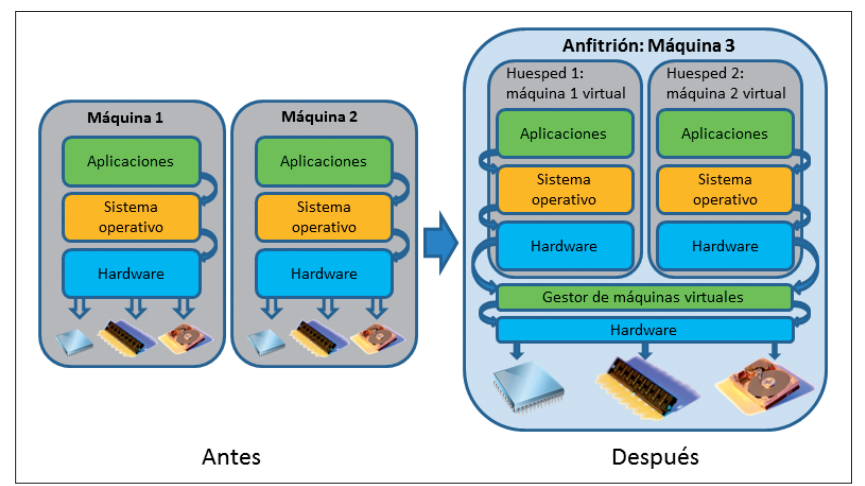

Figura 1. Virtualización de hardware: las máquinas 1 y 2 son virtualizadas y pasan a funcionar en la máquina 3, que actúa como anfitriona. El software de las máquinas 1 y 2 es trasladado intacto, pero ahora utiliza los recursos hardware de la máquina anfitriona.

Si tenemos un conjunto de servidores en nuestra intranet y queremos ejecutarlos en una única máquina, en primer lugar habría que virtualizar cada una de las máquinas físicas con un software específico para servidores (como Xen Server 5.5). Esto nos dará un fichero por cada máquina virtualizada. El siguiente paso será instalar en la máquina anfitriona el mismo software utilizado, que nos permitirá desplegar las máquinas virtualizadas. Hecho esto, únicamente restaría

ejecutar las máquinas virtuales que hemos creado en la máquina anfitriona.

La realización de copias de seguridad es un servicio prestado por cualquier software de virtualización

\section{Virtualización de sistema operativo}

También conocida como virtualización de software, es similar a la virtualización de hardware pero en este caso la máquina anfitriona ejecuta un sistema operativo, y el software de virtualización interactúa contra él. De este modo, independientemente de los sistemas operativos propios de cada máquina virtual, podremos utilizar la máquina anfitriona ya que posee su propio sistema operativo, ya sea personal o de servidor (figura 2). Existe un gran catálogo de software que nos puede ayudar a realizar virtualizaciones de sistema operativo, como Virtuozzo, Solaris Containers y KVM. Por ejemplo, podemos tener una computadora con un sistema operativo Windows 7 ejecutando una máquina virtual con un Ubuntu 10.10. De esta manera podremos ejecutar software de Ubuntu sin tener que cambiar de máquina o sin tener que instalar en la máquina dos sistemas operativos.

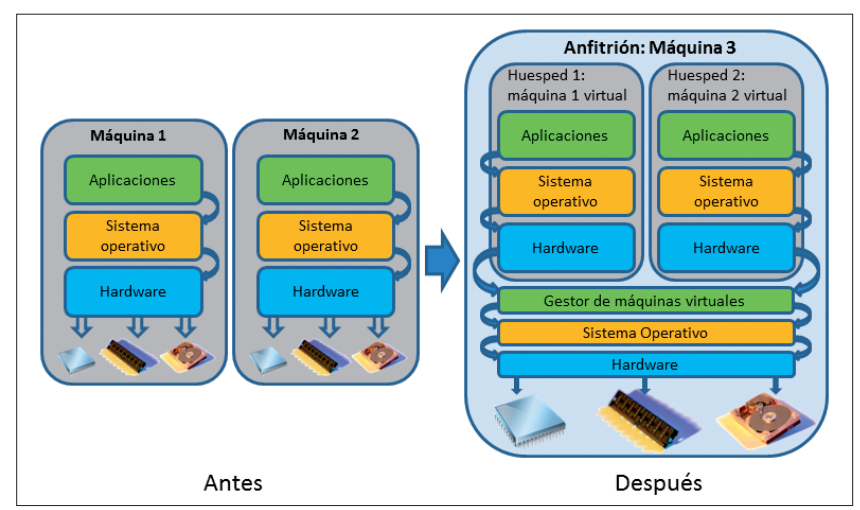

Figura 2. Virtualización de sistema operativo: las máquinas 1 y 2 son virtualizadas y pasan a funcionar en la máquina 3, que actúa como anfitriona. En la máquina 3 puede ejecutarse cualquier software con el sistema operativo anfitrión, o cualquier software con el sistema operativo de las máquinas 1 y 2 mediante las máquinas virtuales.

\section{Virtualización de almacenamiento}

Es la unión de múltiples dispositivos de almacenamiento conectados en red, de tal manera que parezcan una única unidad (figura 3). Es un concepto muy fácil de entender aunque muy complicado de implementar tecnológicamente (SNIA, 2008). Existe un amplio catálogo de software que nos permite hacer virtualización de almacenamiento. Algunos ejemplos son HP StorageWorks, DataCore y EMC Networker.

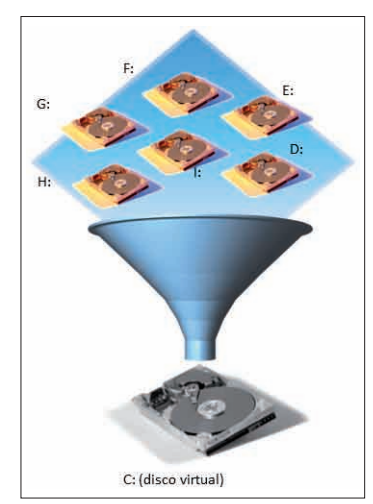

Figura 3. Virtualización de almacenamiento: los discos du$\operatorname{ros} \mathrm{D}, \mathrm{E}, \mathrm{F}, \mathrm{G}, \mathrm{H}$, I son interpretados como una única unidad de almacenamiento virtual, $\mathrm{C}$. 


\section{Virtualización de escritorio}

Consiste en la separación del entorno de escritorio de la máquina física, permitiendo que sea en una máquina servidora de escritorios donde se ejecuten los programas y se realicen los cálculos, mientras que en otra máquina remota se muestra la interfaz gráfica del escritorio con la que el usuario interactúa (ver figura 3). De esta manera conseguimos tener unos clientes "delgados", sin apenas software instalado, ya que sólo funcionan como interfaces para interactuar con los procesos, que están ejecutándose en el servidor.

El software más utilizado para la virtualización de escritorio es Citrix XenDesktop, Microsoft Remote Desktop Services, MokaFive Suite, Sun Virtual Desktop Infrastructure, VMware View, etc.

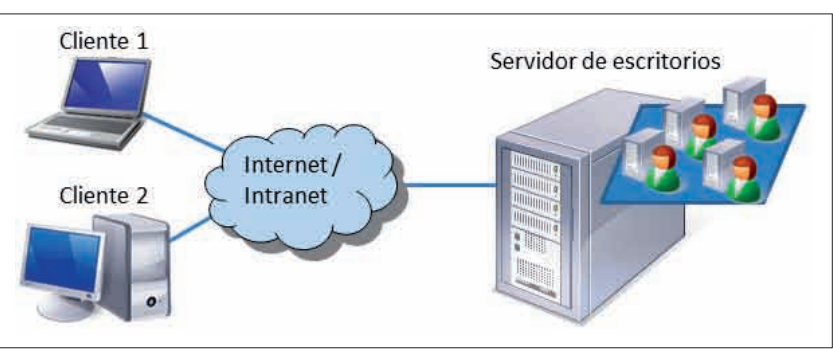

Figura 4. Virtualización de escritorio: los clientes 1 y 2 acceden al servidor de escritorios, que les envía sus respectivos escritorios. Desde el escritorio, los clientes ejecutan los procesos en sus respectivas cuentas en el servidor.

\section{Virtualización de red}

Consiste en poder unir en una red virtual varios elementos de red heterogéneos, ya sean hardware o software, conectados a distintas redes y dispersos topológicamente. Es decir, podemos tener varios elementos de red como computadoras, impresoras, servidores de ficheros, nuestra computadora personal en nuestra casa, etc. localizados en distintos puntos geográficos que, con sólo una conexión a internet, podemos agrupar formando una red virtual, con las ventajas administrativas que ello conlleva (figura 5).

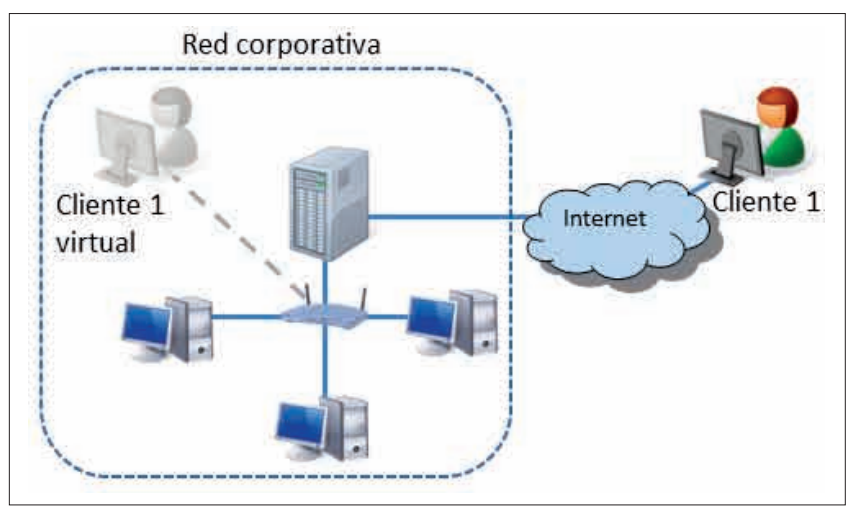

Figura 5. Virtualización de red: el cliente 1 accede a la red corporativa como si estuviese físicamente conectado a ella.

\section{Ventajas e inconvenientes de la virtualización}

La empresa CDW-G analizó en mayo de 2010 el estado de virtualización de agencias federales, estatales y locales en
EUA ( $C D W-G$ 2010). Se hizo una encuesta a más de 600 directivos de empresas de tecnologías de la información y la comunicación (TIC). Según dicho informe, el 77\% de las agencias están implementando al menos una forma de virtualización, y de ese $77 \%$, el $89 \%$ manifiesta obtener beneficios de esta tecnología. Siempre que se desea acometer un proyecto utilizando una tecnología nueva es necesario saber qué ventajas nos ofrece y los inconvenientes que conlleva para saber a qué posibles riesgos nos enfrentamos.

La virtualización reduce el consumo eléctrico y el dióxido de carbono emitido a la atmósfera al reducir el número de máquinas

\section{Ventajas}

- Reducción de costes de hardware: se consigue una reducción de servidores que puede suponer una relación entre 10 a 1 en el caso más optimista a un 6 a 1 en el caso menos optimista. Es decir, que por cada seis máquinas que virtualicemos nos hará falta un solo servidor que las aloje.

- Reducción del consumo eléctrico y el dióxido de carbono emitido a la atmósfera al reducir el número de máquinas.

- Mejora de TCO y ROI: al reducir el número de máquinas estamos disminuyendo el coste total de la propiedad (total cost of ownership, TCO) y por lo tanto conseguiremos un retorno de la inversión (return of investment, ROI) mejor. Si añadimos que estamos ahorrando en la factura eléctrica, el ROI será mejor aun.

- Reducción de los costes de espacio: al tener menos máquinas necesitaremos menos espacio físico para poder desplegar nuestro centro de procesamiento de datos.

- Administración global centralizada y simplificada: son menos máquinas físicas las que hay que administrar, además de que el software de virtualización ayuda a la gestión remota de las máquinas virtuales.

- Rápida incorporación de nuevos recursos para los servidores virtualizados: se trata de una tecnología escalable donde es fácil la incorporación de nuevas máquinas virtuales en una máquina anfitriona.

- Mejora en los procesos de clonación y copia de seguridad del sistema: mayor facilidad para la creación de entornos de prueba que permiten utilizar nuevas aplicaciones sin afectar a la producción en un entorno controlado agilizando el proceso.

- Aislamiento: un fallo general de sistema de una máquina virtual no afecta al resto de máquinas virtuales.

Según un estudio realizado por Network Instruments (2009) después de encuestar a 160 profesionales del sector de las TIC la virtualización también ofrece algunas desventajas:

- Despliegue extensivo: el 55\% de los encuestados ha realizado virtualizaciones de servidores críticos, un $50 \%$ ha virtualizado servidores no críticos como servidores de DNS y DHCP. Sólo el 39\% realizan una virtualización de escritorio.

- Solución de problemas poco claro: el $27 \%$ de los encuestados identifica una falta de visibilidad en la solución de problemas en entornos virtuales. 
- Trampas de la virtualización: el 55\% experimenta más problemas que soluciones, mientras que el $45 \%$ restante piensa que los beneficios superan a los problemas. Esto nos indica que la virtualización, a pesar de ser beneficiosa como hemos visto anteriormente, puede desencadenar más problemas que beneficios si no se sabe implementar adecuadamente.

- Problemas con los administradores de virtualización: el $59 \%$ opina que carecen de experiencia para gestionar adecuadamente la tecnología.

- Costes demasiado altos: el $47 \%$ opina que los costes de despliegue son demasiado altos.

En 2002 Alòs-Moner afirmaba: "para que una intranet tenga éxito son necesarias dos condiciones: el compromiso de la dirección, y que contribuya a la mejora de los procesos". En el caso de la virtualización de una intranet es necesaria exactamente la misma implicación por parte de la dirección y además es necesaria una política de formación y liderazgo por parte de los gestores tal y como concluye el estudio de Network Instruments.

\section{Casos de éxito}

Como hemos visto en los apartados anteriores la virtualización es un concepto que puede ofrecer beneficios si se realiza un buen análisis de nuestras necesidades, se realiza una justa implementación y un correcto mantenimiento. En este apartado vamos a mostrar un conjunto de casos de éxito en la virtualización.

\section{Virtualización en la UC3M}

A mediados de 2009 se inicia un análisis para la virtualización de los servidores del parque informático de la Universidad Carlos III de Madrid gestionado por el Servicio de Informática y Comunicaciones de la misma. Tras un proceso de evaluación, selección y despliegue de la infraestructura, se inicia en el año 2010 un proceso de virtualización de servidores que continúa en la actualidad. A día de hoy se han virtualizado 40 servidores correspondientes a áreas de sistemas, desarrollo, aulas informáticas, audiovisuales, etc. (WebUC3M, 2011).

Paralelamente se llevó a cabo un proyecto cuyo objetivo era poner a disposición de los alumnos equipos virtuales en los que pudieran realizar prácticas, mejorando la calidad del servicio ofreciendo aulas virtuales 24 h (UC3M, 2010). A día de hoy hay virtualizados 120 PCs disponibles para toda la comunidad universitaria.

Se está desplegando una nueva infraestructura de virtualización basada en software libre, cuyo objetivo es proporcionar equipos virtuales a teletrabajadores (UC3M, 2010).

\section{Virtualización de la intranet de la US Navy}

Según informa el artículo de la revista Information week, en 2001 la intranet de la Armada de los Estados Unidos era gestionada por la empresa EDS, empresa de TIC que posteriormente sería una subsidiaria de HP. Por aquel entonces dicha intranet tenía 700.000 usuarios y 40 granjas de servidores repartidos por todo el territorio del país e incluso en varios navíos de guerra. Tras el ataque del 11 de septiembre de 2001 la intranet perdió 30 de sus servidores y el $70 \%$ de la información total de la intranet cuando el Pentágono fue atacado por un avión comercial.

Después de aquel fatídico suceso la empresa EDS se enfrentó al desafío de diseñar una nueva arquitectura robusta ante desastres y reducir el consumo eléctrico de los 2.700 servidores de la intranet que hacía mermar el presupuesto.

EDS consiguió su objetivo reduciendo el consumo en una relación de 9 a 1, pasando de los 2.700 servidores a 300 físicos. Esto supuso un ahorro en la factura de energía eléctrica de 1,6 millones de dólares anuales (Babcock, 2008).

\section{Virtualización en Chesapeake, VA, EUA}

El calor producido por los servidores del centro de datos de la ciudad de Chesapeake, Virginia, estaba reduciendo la vida útil de los equipos que albergaba. En lugar de ampliar el centro de datos y volver a tener servidores infrautilizados, la ciudad decidió desplegar 84 servidores virtuales reduciendo significativamente su proliferación y aumentando la longevidad de su hardware.

El proyecto acabó en agosto de 2010 y la ciudad consiguió una reducción en gasto energético de 36.000 dólares anuales (un $50 \%$ menos con respecto al año anterior), se redujo en 200.000 dólares el gasto en hardware anual y se pasó de tener 135 servidores físicos a 20 (un ratio de 6,75 a 1).

El ahorro conseguido por la ciudad de Chesapeake le ha permitido trabajar en otros proyectos que de otra manera no habrían podido ser financiados. Además gracias a la virtualización han conseguido un sistema de información robusto que incluye backups distribuidos para poder recuperarse en caso de desastres (CDW-G, 2010).

\section{Virtualización en McKinney, TX, EUA}

Mckinney es una de las 10 ciudades con mayor crecimiento en los EUA según la revista Forbes, y el centro de datos de la ciudad ha sufrido el mismo nivel crecimiento. Para poder hacer frente al mismo la ciudad se embarcó en una iniciativa de que duró tres años y que concluyó en diciembre de 2009 con 120 de sus 158 servidores virtualizados, es decir, un $76 \%$ de virtualización.

Se acortó el tiempo de instalación de servidores de varios días a horas y mejoró notablemente la productividad de plantilla liberando tiempo para realizar otras tareas.

El tiempo y el dinero ahorrados han permitido a la ciudad de McKinney acometer un proyecto de virtualización de escritorio, en la actualidad en elaboración, que permitirá doblar el tiempo de vida del hardware y ayudará a la plantilla de TIC a instalar nuevas aplicaciones y actualizaciones de forma centralizada, mejorando la experiencia del usuario final (CDW-G, 2010).

\section{Virtualización del sistema de emergencias Stanly county}

Antes de la virtualización el condado de Stanly, North Carolina, EUA, no tenía una estrategia de copias de seguridad para su centro de llamadas de emergencia, el cual está en el sótano del palacio de justicia del condado. Cualquier desastre podría interrumpir las comunicaciones de emergencia. Esta posibilidad así como la falta de espacio para más servidores 
físicos, el ahorro de energía y refrigerado del centro de datos fueron los motivos por los que se tomó la decisión de virtualizar sus servidores.

Tras ello se consiguió un centro de datos con la información redundante, se mejoró la disponibilidad al ciudadano del sistema de llamadas de emergencia y se condensaron siete servidores físicos en tres.

Debido al éxito en este primer proyecto el condado de Stanly va a continuar con la virtualización de su red. Chad Coble, director de TIC del condado, recomienda que los servidores virtuales estén enlazados con sistemas de almacenamiento externos para poder salvaguardar toda la información (CDW-G, 2010).

\section{Virtualización en Skokie}

Tras una fuerte tormenta el 24 de julio de 2010 el pueblo de Skokie, en Illinois, EUA, sufrió una gran inundación que dejó anegados los sistemas de información del pueblo. El equipo de TIC necesitó muchos días de trabajo para volver a restaurar los sistemas de información de la ciudad.

Durante una fase de actualización de los sistemas informáticos decidieron mejorarlos y afrontar un cambio hacia la virtualización del almacenamiento para poder recuperarse ante desastres en el futuro.

Con la virtualización, además de aportar una estrategia de recuperación ante desastres, el pueblo de Skokie ha conseguido reducir el consumo de energía y la generación de calor reemplazando doce servidores por cuatro servidores energéticamente más eficientes y cuatro nodos SAN (storage area network). En definitiva, se ha mejorado el servicio a los empleados y a los ciudadanos, se ha conseguido un importante ahorro en tiempo de la plantilla de TIC y el centro de datos se ha alineado con iniciativas ecologistas del pueblo de Skokie.

El próximo proyecto que va a afrontar el pueblo de Skokie será la construcción de una nueva comisaría, y se pretende realizar una virtualización de datos entre las comisarías del pueblo (CDW-G, 2010)

\section{Virtualización de los Centros de datos gestionados de Telefónica}

La división de la compañía Telefónica que ofrece soluciones de TIC para albergar y administrar sistemas y aplicaciones de negocio, Telefónica España Grandes Empresas, entró en el mundo de la virtualización de sistemas con el objetivo de acercarse al concepto de infraestructuras privadas virtuales y proporcionar a sus clientes entornos virtuales sobre plataformas de gran tamaño desplegadas en sus centros de datos gestionados (CDGs). Estos centros se dedican al alojamiento y gestión de sistemas y aplicaciones de negocio de los clientes. En concreto, uno de los productos que Telefónica ofrece es la gestión de sus centros de procesamiento de datos (CPD). En 2007, antes de la virtualización, se debía gestionar cada uno de los CPDs de los clientes por separado, teniendo al menos un servidor dedicado por cada cliente. Después de la virtualización en 2008, todos los CPDs corren sobre servidores compartidos. Los beneficios resultantes de esta virtualización fueron (VMware, 2008; Virtualización.TV, 2011a; De-Lima, 2008):
- reducción de entre un $20 \%$ y un $30 \%$ del coste total del servicio a los clientes;

- cambio en el modelo de pago que evita la necesidad de realizar inversiones en equipamiento. Es decir, el cliente no tiene que pagar los servidores;

- obtención de un elevado grado de aislamiento e independencia entre servidores de distintos clientes mientras comparten una misma infraestructura física;

- mejora de la calidad del servicio al cliente proporcionando servidores de mayor disponibilidad que en entornos físicos antes de la virtualización;

- optimización del uso de los recursos físicos de la plataforma de virtualización;

- flexibilización de las infraestructuras que ofrecen en su centro de datos gestionados;

- Ahorro significativo en espacio físico, consumo eléctrico y refrigeración.

El proceso de virtualización requiere un plan de formación de la plantilla, debido a que supone un cambio de paradigma en la arquitectura software

\section{Virtualización del laboratorio de pruebas de IBM}

El grupo de desarrollo IBM Lotus crea y prueba software que permite a las empresas comunicarse y colaborar de forma eficiente. En 2006, uno de los laboratorios de la compañía se tuvo que enfrentar a un gran desafío: muchos servidores tenían muchos sistemas operativos instalados sobre un mismo hardware, lo que provocaba problemas de seguridad. Este laboratorio necesita ejecutar pruebas de software en distintas máquinas con distintos sistemas operativos, almacenar el estado de las pruebas y restaurar el sistema de cada servidor a un estado limpio. Elaborar un sistema complejo de pruebas suponía muchas horas de trabajo que además era muy poco reutilizable. Por último, replicar entornos exactos de pruebas era muy complicado.

En un esfuerzo para mejorar la eficiencia en los procesos de pruebas, IBM desplegó un sistema de virtualización de los servidores de su laboratorio. De esta manera los desarrolladores pueden capturar el estado completo de un entorno de pruebas y redesplegar dichas pruebas en otra máquina exactamente en el mismo estado. Los resultados que el grupo consiguió fueron (VMware, 2007; Virtualización.TV, 2011b):

- reducción del número de servidores necesarios a sólo el $10 \%$, donde 10 máquinas virtuales se ejecutan en un sólo servidor físico;

- disminución del tiempo empleado en la ejecución de pruebas de ocho horas a apenas unos minutos, lo que supone un ahorro de dos horas por persona y prueba;

- reducción de costes al retirar los servidores viejos y comprar sólo los necesarios;

- ahorro significativo en electricidad tanto para los servidores como para su refrigeración. Ahorro también en el mantenimiento en los equipos. 


\section{Conclusiones}

La virtualización ha demostrado ser un mecanismo muy útil para implementar una arquitectura de servidores, abaratando los costes en hardware, gestión y demanda eléctrica. La reducción en hardware puede ser de 6 servidores a 1 en el caso más pesimista a 10 servidores a 1 en el caso más optimista. En general son numerosas sus ventajas y factible su implantación, como demuestran los casos de éxito, pero también existen inconvenientes. Uno de los problemas es que para poder acometer un proyecto de virtualización es necesario un plan de formación de la plantilla, debido a que supone un cambio de paradigma en la arquitectura software y es necesario que conozca el entorno físico donde se está trabajando para minimizar los problemas. La virtualización es un proceso que se debe acometer de forma iterativa, planificando las distintas fases en función de las dependencias entre servicios y su criticidad, e ir consolidándolas en el tiempo. Los costes y riesgos asociados deben evaluarse para obtener un retorno de la inversión en el plazo previsto. El software utilizado para la virtualización debe ser también objeto de estudio, ya que se trata de una tecnología clave para obtener una intranet eficiente, estable, escalable y sostenible, tanto desde su diseño inicial como en proyectos de mejora de una intranet ya existente.

La virtualización es útil para implementar una arquitectura de servidores, abaratando costes en hardware, gestión y demanda eléctrica

\section{Bibliografía}

Alòs-Moner, Adela. "Intranets: sonrisas y lágrimas". El profesional de la información, 2002, v. 11, n. 1, pp. 4-8. http://www.elprofesionaldelainformacion.com/conteni dos/2002/enero/1.pdf

Babcock, Charles. "U.S. Navy intranet realizes big savings through virtualization". Informtion week: the business value of technology, 10 Oct. 2008.

http://www.informationweek.com/news/hardware/data_ centers/showArticle.jhtm/?articleID=211100206

CDW-G. "Government virtualization report 2010". http://newsroom.cdwg.com/features/feature-07-1310.html

De-Lima, Amaya. "El centro de datos gestionados de Telefónica adopta la virtualización”, TCN, sept. 2008.

http://www.revistatcn.com/id960/el-centro-de-datos-ges tionados-de-telefonica-adopta-la-virtualizacion/

Fundación Vida Sostenible, El precio de la factura eléctrica: tarifas (Diciembre 2010).

http://www.vidasostenible.org/observatorio/f2_final. asp?idinforme $=1391$

Gartner Inc., Gartner identifies the top 10 strategic technologies for 2010, 2010.

http://www.gartner.com/it/page.jsp?id=1210613
Networks Instruments, "Interop snapshot: virtualization deployments and challenges top of mind for attendee", 2009.

Popek, Gerald J.; Goldberg, Robert. "Formal requirements for virtualizable third generation architectures", Communications of the ACM, 1974, v. 17, n. 7, pp. 412-421.

http://www.cs.berkeley.edu/ brewer/cs262/VM-require ments.pdf

Red Eléctrica de España, Detalle de la estructura de generación en tiempo real.

https://demanda.ree.es/generacion_acumulada.html

SNIA Green Storage Initiative. Best practices for energy efficient storage operations, 2008.

Turban, Efrain; King, Dave; Lee, Jae Kyu; Viehland, Dennis. "Building ecommerce applications and infrastructure". Electronic commerce: a managerial perspective (5th ed.). Prentice-Hall, 2008, $27 \mathrm{pp}$.

http://wps.prenhall.com/wps/media/objects/5073/ 5195381/pdf/Online_Chapter_19.pdf

UC3M, Implantacion del servicio de teletrabajo sobre una plataforma de virtualizacion de escritorios. CAU-UC3M, 2010.

http://www.uc3m.es/portal/page/portal/informatica/CAU/ Servicios/Soporte_Equipos_Personales/Resumen\%202\%BA \%20Piloto\%20de\%20Teletrabajo.pdf

UC3M. Nuevo servicio Aula Virtual 24 horas, 2010.

http://www.uc3m.es/portal/page/portal/informatica/Noti cias_Sdiario/AULA_VIRTUAL_24_HORAS

UC3M. Virtualización de los servidores de impresión, 2011. http://www.uc3m.es/portal/page/portal/informatica/Noti cias_Sdiario/Virtualizacion_servidores_Impresion

Virtualizacion.tv. Caso de éxito de Telefónica, febrero 2011 a.

http://www.virtualizacion.tv/2011/02/21/episodio-7-casoexito-telefnica-vca4-desktop-nueva-certificacin-vmwarevkernel.html

Virtualizacion.tv. El caso de éxito de IBM, abril 2011b. http://www.virtualizacion.tv/2011/04/04/episodio-13-vir tualizaciontv-itunes-store-caso-exito-ibm-foros-virtualiza cion-espanol.html

VMware Inc. How VMware virtualization right-sizes IT infrastructure to reduce power consumption, 2008a http://www.vmware.com/files/pdf/WhitePaper_Reduce PowerConsumption.pdf

VMWare Inc. Reduce energy costs and go green with VMWare Green IT Solutions, 2010.

http://www.vmware.com/files/pdf/VMware-GREEN-ITOVERVIEW-SB-EN.pdf

http://www.vmware.com/files/pdf/green_solutionbrief.pdf

VMware Inc. WMware customer snapshot: IBM, 2007.

http://www.vmware.com/files/pdf/customers/07Q2_ss_ $v m w \_i b m \_e n g l i s h . p d f$

VMware Inc. WMware customer snapshot: Telefónica, 2008b.

http://www.vmware.com/files/es/pdf/08Q2_cs_vmw_tele fonica_spanish.pdf 


\section{Rapidez, precisión, flexibilidad}

En el siglo XXI, más que nunca, la información es poder. Ayudar a empresas e instituciones a gestionar y rentabilizar su caudal informativo, tanto el que poseen como el que genera diariamente su actividad, es el objetivo y la razón de ser de Baratz.

\section{www.baratz.es}

\section{0 informa@baratz.es}

